\title{
A Viscosity of Cesàro Mean Approximation Methods for a Mixed Equilibrium, Variational Inequalities, and Fixed Point Problems
}

\section{Thanyarat Jitpeera, Phayap Katchang, and Poom Kumam}

Department of Mathematics, Faculty of Science, King Mongkut's University of Technology Thonburi (KMUTT), Bangmod, Bangkok 10140, Thailand

Correspondence should be addressed to Poom Kumam, poom.kum@kmutt.ac.th

Received 6 September 2010; Accepted 15 October 2010

Academic Editor: Qamrul Hasan Ansari

Copyright (c) 2011 Thanyarat Jitpeera et al. This is an open access article distributed under the Creative Commons Attribution License, which permits unrestricted use, distribution, and reproduction in any medium, provided the original work is properly cited.

\begin{abstract}
We introduce a new iterative method for finding a common element of the set of solutions for mixed equilibrium problem, the set of solutions of the variational inequality for a $\beta$-inversestrongly monotone mapping, and the set of fixed points of a family of finitely nonexpansive mappings in a real Hilbert space by using the viscosity and Cesaro mean approximation method. We prove that the sequence converges strongly to a common element of the above three sets under some mind conditions. Our results improve and extend the corresponding results of Kumam and Katchang (2009), Peng and Yao (2009), Shimizu and Takahashi (1997), and some authors.
\end{abstract}

\section{Introduction}

Throughout this paper, we assume that $H$ is a real Hilbert space with inner product and norm are denoted by $\langle\cdot, \cdot\rangle$ and $\|\cdot\|$, respectively and let $C$ be a nonempty closed convex subset of $H$. A mapping $T: C \rightarrow C$ is called nonexpansive if $\|T x-T y\| \leq\|x-y\|$, for all $x, y \in C$. We use $F(T)$ to denote the set of fixed points of $T$, that is, $F(T)=\{x \in C: T x=x\}$. It is assumed throughout the paper that $T$ is a nonexpansive mapping such that $F(T) \neq \emptyset$. Recall that a self-mapping $f: C \rightarrow C$ is a contraction on $C$ if there exists a constant $\alpha \in[0,1)$ and $x, y \in C$ such that $\|f(x)-f(y)\| \leq \alpha\|x-y\|$.

Let $\varphi: C \rightarrow \mathbb{R} \cup\{+\infty\}$ be a proper extended real-valued function and $\phi$ be a bifunction of $C \times C$ into $\mathbb{R}$, where $\mathbb{R}$ is the set of real numbers. Ceng and Yao [1] considered the following mixed equilibrium problem for finding $x \in C$ such that

$$
\phi(x, y)+\varphi(y) \geq \varphi(x), \quad \forall y \in C .
$$


The set of solutions of $(1.1)$ is denoted by $\operatorname{MEP}(\phi, \varphi)$. We see that $x$ is a solution of problem (1.1) implies that $x \in \operatorname{dom} \varphi=\{x \in C \mid \varphi(x)<+\infty\}$. If $\varphi=0$, then the mixed equilibrium problem (1.1) becomes the following equilibrium problem is to find $x \in C$ such that

$$
\phi(x, y) \geq 0, \quad \forall y \in C \text {. }
$$

The set of solutions of (1.2) is denoted by $\operatorname{EP}(\phi)$. The mixed equilibrium problems include fixed point problems, variational inequality problems, optimization problems, Nash equilibrium problems, and the equilibrium problem as special cases. Numerous problems in physics, optimization, and economics reduce to find a solution of (1.2). Some methods have been proposed to solve the equilibrium problem (see [2-14]).

Let $B: C \rightarrow H$ be a mapping. The variational inequality problem, denoted by $\operatorname{VI}(C, B)$, is to find $x \in C$ such that

$$
\langle B x, y-x\rangle \geq 0
$$

for all $y \in C$. The variational inequality problem has been extensively studied in the literature. See, for example, $[15,16]$ and the references therein. A mapping $B$ of $C$ into $H$ is called monotone if

$$
\langle B x-B y, x-y\rangle \geq 0,
$$

for all $x, y \in C$. $B$ is called $\beta$-inverse-strongly monotone if there exists a positive real number $\beta>0$ such that for all $x, y \in C$

$$
\langle B x-B y, x-y\rangle \geq \beta\|B x-B y\|^{2} .
$$

Let $A$ be a strongly positive linear bounded operator on $H$ : that is, there is a constant $\bar{\gamma}>0$ with property

$$
\langle A x, x\rangle \geq \bar{\gamma}\|x\|^{2}, \quad \forall x \in H .
$$

A typical problem is to minimize a quadratic function over the set of the fixed points of a nonexpansive mapping on a real Hilbert space $H$ :

$$
\min _{x \in F(T)} \frac{1}{2}\langle A x, x\rangle-h(x)
$$

where $A$ is strongly positive linear bounded operator and $h$ is a potential function for $\gamma f$ (i.e., $h^{\prime}(x)=\gamma f(x)$ for $\left.x \in H\right)$. Moreover, it is shown in [17] that the sequence $\left\{x_{n}\right\}$ defined by the scheme

$$
x_{n+1}=\epsilon_{n} \gamma f\left(x_{n}\right)+\left(1-\epsilon_{n} A\right) T x_{n},
$$

converges strongly to $z=P_{F(T)}(I-A+\gamma f)(z)$. 
In 1997, Shimizu and Takahashi [18] originally studied the convergence of an iteration process $\left\{x_{n}\right\}$ for a family of nonexpansive mappings in the framework of a real Hilbert space. They restate the sequence $\left\{x_{n}\right\}$ as follows:

$$
x_{n+1}=\alpha_{n} x+\left(1-\alpha_{n}\right) \frac{1}{n+1} \sum_{j=0}^{n} T^{j} x_{n}, \quad \text { for } n=0,1,2, \ldots,
$$

where $x_{0}$ and $x$ are all elements of $C$ and $\alpha_{n}$ is an appropriate in $[0,1]$. They proved that $\left\{x_{n}\right\}$ converges strongly to an element of fixed point of $T$ which is the nearest to $x$.

In 2007, Plubtieng and Punpaeng [19] proposed the following iterative algorithm:

$$
\begin{gathered}
\phi\left(u_{n}, y\right)+\frac{1}{r_{n}}\left\langle y-u_{n}, u_{n}-x_{n}\right\rangle \geq 0, \quad \forall y \in H, \\
x_{n+1}=\epsilon_{n} \gamma f\left(x_{n}\right)+\left(I-\epsilon_{n} A\right) T u_{n} .
\end{gathered}
$$

They proved that if the sequence $\left\{\epsilon_{n}\right\}$ and $\left\{r_{n}\right\}$ of parameters satisfy appropriate condition, then the sequences $\left\{x_{n}\right\}$ and $\left\{u_{n}\right\}$ both converge to the unique solution $z$ of the variational inequality

$$
\langle(A-\gamma f) z, x-z\rangle \geq 0, \quad \forall x \in F(T) \cap \operatorname{EP}(\phi)
$$

which is the optimality condition for the minimization problem

$$
\min _{x \in F(T) \cap \mathrm{EP}(\phi)} \frac{1}{2}\langle A x, x\rangle-h(x),
$$

where $h$ is a potential function for $\gamma f$ (i.e., $h^{\prime}(x)=\gamma f(x)$ for $x \in H$ ).

In 2008, Peng and Yao [20] introduced an iterative algorithm based on extragradient method which solves the problem of finding a common element of the set of solutions of a mixed equilibrium problem, the set of fixed points of a family of finitely nonexpansive mappings and the set of the variational inequality for a monotone, Lipschitz continuous mapping in a real Hilbert space. The sequences generated by $v \in C$,

$$
\begin{gathered}
x_{1}=x \in C, \\
\phi\left(u_{n}, y\right)+\varphi(y)-\varphi\left(u_{n}\right)+\frac{1}{r_{n}}\left\langle y-u_{n}, u_{n}-x_{n}\right\rangle \geq 0, \quad \forall y \in C, \\
y_{n}=P_{C}\left(u_{n}-\gamma_{n} B u_{n}\right), \\
x_{n+1}=\alpha_{n} v+\beta_{n} x_{n}+\left(1-\alpha_{n}-\beta_{n}\right) W_{n} P_{C}\left(u_{n}-\lambda_{n} B y_{n}\right),
\end{gathered}
$$

for all $n \geq 1$, where $W_{n}$ is $W$-mapping. They proved the strong convergence theorems under some mind conditions.

In this paper, motivated by the above results and the iterative schemes considered in $[9,18-20]$, we introduce a new iterative process below based on viscosity and Cesàro mean 
approximation method for finding a common element of the set of fixed points of a family of finitely nonexpansive mappings, the set of solutions of the variational inequality problem for a $\beta$-inverse-strongly monotone mapping and the set of solutions of a mixed equilibrium problem in a real Hilbert space. Then, we prove strong convergence theorems which are connected with [5, 21-24]. We extend and improve the corresponding results of Kumam and Katchang [9], Peng and Yao [20], Shimizu and Takahashi [18] and some authors.

\section{Preliminaries}

Let $H$ be a real Hilbert space with inner product $\langle\cdot, \cdot\rangle$ and norm $\|\cdot\|$ and let $C$ be a nonempty closed convex subset of $H$. Then

$$
\begin{gathered}
\|x-y\|^{2}=\|x\|^{2}-\|y\|^{2}-2\langle x-y, y\rangle \\
\|\lambda x+(1-\lambda) y\|^{2}=\lambda\|x\|^{2}+(1-\lambda)\|y\|^{2}-\lambda(1-\lambda)\|x-y\|^{2}
\end{gathered}
$$

for all $x, y \in H$ and $\lambda \in[0,1]$. For every point $x \in H$, there exists a unique nearest point in $C$, denoted by $P_{C} x$, such that

$$
\left\|x-P_{C} x\right\| \leq\|x-y\|, \quad \forall y \in C
$$

$P_{C}$ is called the metric projection of $H$ onto $C$. It is well known that $P_{C}$ is a nonexpansive mapping of $H$ onto $C$ and satisfies

$$
\left\langle x-y, P_{C} x-P_{C} y\right\rangle \geq\left\|P_{C} x-P_{C} y\right\|^{2},
$$

for every $x, y \in H$. Moreover, $P_{C} x$ is characterized by the following properties: $P_{C} x \in C$ and

$$
\begin{gathered}
\left\langle x-P_{C} x, y-P_{C} x\right\rangle \leq 0, \\
\|x-y\|^{2} \geq\left\|x-P_{C} x\right\|^{2}+\left\|y-P_{C} x\right\|^{2},
\end{gathered}
$$

for all $x \in H, y \in C$. Let $B$ be a monotone mapping of $C$ into $H$. In the context of the variational inequality problem the characterization of projection (2.5) implies the following:

$$
u \in \mathrm{VI}(C, B) \Longleftrightarrow u=P_{C}(u-\lambda B u), \quad \lambda>0
$$

It is also known that $H$ satisfies the Opial condition [25], that is, for any sequence $\left\{x_{n}\right\} \subset H$ with $x_{n} \rightarrow x$, the inequality

$$
\liminf _{n \rightarrow \infty}\left\|x_{n}-x\right\|<\liminf _{n \rightarrow \infty}\left\|x_{n}-y\right\|
$$

holds for every $y \in H$ with $x \neq y$. 
A set-valued mapping $U: H \rightarrow 2^{H}$ is called monotone if for all $x, y \in H, f \in U x$ and $g \in U y$ imply $\langle x-y, f-g\rangle \geq 0$. A monotone mapping $U: H \rightarrow 2^{H}$ is maximal if the graph of $G(U)$ of $U$ is not properly contained in the graph of any other monotone mapping. It is known that a monotone mapping $U$ is maximal if and only if for $(x, f) \in H \times H,\langle x-y, f-g\rangle \geq 0$ for every $(y, g) \in G(U)$ implies $f \in U x$. Let $B$ be a monotone mapping of $C$ into $H$ and let $N_{C} \bar{y}$ be the normal cone to $C$ at $\bar{y} \in C$, that is, $N_{C} \bar{y}=\{w \in H:\langle u-\bar{y}, w\rangle \leq 0, \forall u \in C\}$ and define

$$
U \bar{y}= \begin{cases}B \bar{y}+N_{C} \bar{y}, & \bar{y} \in C, \\ \emptyset, & \bar{y} \notin C .\end{cases}
$$

Then $U$ is the maximal monotone and $0 \in U \bar{y}$ if and only if $\bar{y} \in \mathrm{VI}(C, B)$; see [26].

For solving the mixed equilibrium problem, let us give the following assumptions for a bifunction $\phi: C \times C \rightarrow \mathbb{R}$ and a proper extended real-valued function $\varphi: C \rightarrow \mathbb{R} \cup\{+\infty\}$ satisfies the following conditions:

(A1) $\phi(x, x)=0$ for all $x \in C$;

(A2) $\phi$ is monotone, that is, $\phi(x, y)+\phi(y, x) \leq 0$ for all $x, y \in C$;

(A3) for each $x, y, z \in C, \lim _{t \rightarrow 0} \phi(t z+(1-t) x, y) \leq \phi(x, y)$;

(A4) for each $x \in C, y \mapsto \phi(x, y)$ is convex and lower semicontinuous;

(A5) for each $y \in C, x \mapsto \phi(x, y)$ is weakly upper semicontinuous;

(B1) for each $x \in H$ and $r>0$, there exist a bounded subset $D_{x} \subseteq C$ and $y_{x} \in C$ such that for any $z \in C \backslash D_{x}$,

$$
\phi\left(z, y_{x}\right)+\varphi\left(y_{x}\right)+\frac{1}{r}\left\langle y_{x}-z, z-x\right\rangle<\varphi(z)
$$

(B2) $C$ is a bounded set.

We need the following lemmas for proving our main results.

Lemma 2.1 (Peng and Yao [20]). Let $C$ be a nonempty closed convex subset of $H$. Let $\phi: C \times C \rightarrow \mathbb{R}$ be a bifunction satisfies (A1)-(A5) and let $\varphi: C \rightarrow \mathbb{R} \cup\{+\infty\}$ be a proper lower semicontinuous and convex function. Assume that either (B1) or (B2) holds. For $r>0$ and $x \in H$, define a mapping $T_{r}: H \rightarrow C$ as follows:

$$
T_{r}(x)=\left\{z \in C: \phi(z, y)+\varphi(y)+\frac{1}{r}\langle y-z, z-x\rangle \geq \varphi(z), \quad \forall y \in C\right\}
$$

for all $x \in H$. Then, the following hold.

(1) For each $x \in H, T_{r}(x) \neq \emptyset$;

(2) $T_{r}$ is single-valued;

(3) $T_{r}$ is firmly nonexpansive, that is, for any $x, y \in H,\left\|T_{r} x-T_{r} y\right\|^{2} \leq\left\langle T_{r} x-T_{r} y, x-y\right\rangle$;

(4) $F\left(T_{r}\right)=\operatorname{MEP}(\phi, \varphi)$;

(5) $\operatorname{MEP}(\phi, \varphi)$ is closed and convex. 
Lemma 2.2 (Xu [27]). Assume $\left\{a_{n}\right\}$ is a sequence of nonnegative real numbers such that

$$
a_{n+1} \leq\left(1-\alpha_{n}\right) a_{n}+\delta_{n}, \quad n \geq 0,
$$

where $\left\{\alpha_{n}\right\}$ is a sequence in $(0,1)$ and $\left\{\delta_{n}\right\}$ is a sequence in $\mathbb{R}$ such that

(1) $\sum_{n=1}^{\infty} \alpha_{n}=\infty$,

(2) $\lim \sup _{n \rightarrow \infty}\left(\delta_{n} / \alpha_{n}\right) \leq 0$ or $\sum_{n=1}^{\infty}\left|\delta_{n}\right|<\infty$.

Then $\lim _{n \rightarrow \infty} a_{n}=0$.

Lemma 2.3 (Osilike and Igbokwe [28]). Let $(C,\langle\cdot, \cdot\rangle)$ be an inner product space. Then for all $x, y, z \in C$ and $\alpha, \beta, \gamma \in[0,1]$ with $\alpha+\beta+\gamma=1$, we have

$$
\|\alpha x+\beta y+\gamma z\|^{2}=\alpha\|x\|^{2}+\beta\|y\|^{2}+\gamma\|z\|^{2}-\alpha \beta\|x-y\|^{2}-\alpha \gamma\|x-z\|^{2}-\beta \gamma\|y-z\|^{2} .
$$

Lemma 2.4 (Suzuki [29]). Let $\left\{x_{n}\right\}$ and $\left\{y_{n}\right\}$ be bounded sequences in a Banach space $X$ and let $\left\{\beta_{n}\right\}$ be a sequence in $[0,1]$ with $0<\lim _{\inf _{n \rightarrow \infty}} \beta_{n} \leq \limsup _{n \rightarrow \infty} \beta_{n}<1$. Suppose $x_{n+1}=$ $\left(1-\beta_{n}\right) y_{n}+\beta_{n} x_{n}$ for all integers $n \geq 0$ and $\lim \sup _{n \rightarrow \infty}\left(\left\|y_{n+1}-y_{n}\right\|-\left\|x_{n+1}-x_{n}\right\|\right) \leq 0$. Then, $\lim _{n \rightarrow \infty}\left\|y_{n}-x_{n}\right\|=0$.

Lemma 2.5 (Marino and $\mathrm{Xu}$ [17]). Assume $A$ is a strongly positive linear bounded operator on a Hilbert space $H$ with coefficient $\bar{\gamma}>0$ and $0<\rho \leq\|A\|^{-1}$. Then $\|I-\rho A\| \leq 1-\rho \bar{\gamma}$.

Lemma 2.6 (Bruck [30]). Let $C$ be a nonempty bounded closed convex subset of a uniformly convex Banach space $E$ and $T: C \rightarrow C$ a nonexpansive mapping. For each $x \in C$ and the Cesàro means $T_{n} x=(1 /(n+1)) \sum_{i=0}^{n} T^{i} x$, then $\lim \sup _{n \rightarrow \infty}\left\|T_{n} x-T\left(T_{n} x\right)\right\|=0$.

\section{Main Results}

In this section, we show a strong convergence theorem for finding a common element of the set of fixed points of a family of finitely nonexpansive mappings, the set of solutions of mixed equilibrium problem and the set of solutions of a variational inequality problem for a $\beta$-inverse-strongly monotone mapping in a real Hilbert space by using the viscosity of Cesàro mean approximation method.

Theorem 3.1. Let $C$ be a nonempty closed convex subset of a real Hilbert space $H$. Let $\phi$ be a bifunction of $C \times C$ into real numbers $\mathbb{R}$ satisfying (A1)-(A5) and let $\varphi: C \rightarrow \mathbb{R} \cup\{+\infty\}$ be a proper lower semicontinuos and convex function. Let $T^{i}: C \rightarrow C$ be a nonexpansive mappings for all $i=1,2,3, \ldots, n$, such that $\Theta:=\bigcap_{i=1}^{n} F\left(T^{i}\right) \cap \operatorname{VI}(C, B) \cap \operatorname{MEP}(\phi, \varphi) \neq \emptyset$. Let $f$ be a contraction of $C$ 
into itself with coefficient $\alpha \in(0,1)$ and let $B$ be a $\beta$-inverse-strongly monotone mapping of $C$ into $H$. Let $A$ be a strongly positive bounded linear self-adjoint on $H$ with coefficient $\bar{\gamma}>0$ and $0<\gamma<\bar{\gamma} / \alpha$. Assume that either $B_{1}$ or $B_{2}$ holds. Let $\left\{x_{n}\right\},\left\{y_{n}\right\}$ and $\left\{u_{n}\right\}$ be sequences generated by $x_{0} \in C, u_{n} \in C$ and

$$
\begin{gathered}
\phi\left(u_{n}, y\right)+\varphi(y)-\varphi\left(u_{n}\right)+\frac{1}{r_{n}}\left\langle y-u_{n}, u_{n}-x_{n}\right\rangle \geq 0, \quad \forall y \in C, \\
y_{n}=\delta_{n} u_{n}+\left(1-\delta_{n}\right) P_{C}\left(u_{n}-\lambda_{n} B u_{n}\right), \\
x_{n+1}=\alpha_{n} \gamma f\left(x_{n}\right)+\beta_{n} x_{n}+\left(\left(1-\beta_{n}\right) I-\alpha_{n} A\right) \frac{1}{n+1} \sum_{i=0}^{n} T^{i} y_{n}, \quad \forall n \geq 0,
\end{gathered}
$$

where $\left\{\alpha_{n}\right\},\left\{\beta_{n}\right\}$ and $\delta_{n} \subset(0,1),\left\{\lambda_{n}\right\} \subset(0,2 \beta)$ and $\left\{r_{n}\right\} \subset(0, \infty)$ satisfy the following conditions:

(i) $\sum_{n=0}^{\infty} \alpha_{n}=\infty$ and $\lim _{n \rightarrow \infty} \alpha_{n}=0$,

(ii) $\lim _{n \rightarrow \infty} \delta_{n}=0$,

(iii) $0<\lim \inf _{n \rightarrow \infty} \beta_{n} \leq \lim \sup _{n \rightarrow \infty} \beta_{n}<1$,

(iv) $\left\{\lambda_{n}\right\} \subset[a, b], \exists a, b \in(0,2 \beta)$ and $\lim _{n \rightarrow \infty}\left|\lambda_{n+1}-\lambda_{n}\right|=0$,

(v) $\lim \inf _{n \rightarrow \infty} r_{n}>0$ and $\lim _{n \rightarrow \infty}\left|r_{n+1}-r_{n}\right|=0$.

Then, $\left\{x_{n}\right\}$ converges strongly to $z \in \Theta$, where $z=P_{\Theta}(I-A+\gamma f)(z)$, which is the unique solution of the variational inequality

$$
\langle(A-\gamma f) z, x-z\rangle \geq 0, \quad \forall x \in \Theta
$$

Proof. Now, we have $\left\|\left(1-\beta_{n}\right) I-\alpha_{n} A\right\| \leq 1-\beta_{n}-\alpha_{n} \bar{\gamma}$ (see [9, page 479]). Since $\lambda_{n} \in(0,2 \beta)$ and $B$ is a $\beta$-inverse-strongly monotone mapping. For any $x, y \in C$, we have

$$
\begin{aligned}
\left\|\left(I-\lambda_{n} B\right) x-\left(I-\lambda_{n} B\right) y\right\|^{2} & =\left\|(x-y)-\lambda_{n}(B x-B y)\right\|^{2} \\
& =\|x-y\|^{2}-2 \lambda_{n}\langle x-y, B x-B y\rangle+\lambda_{n}^{2}\|B x-B y\|^{2} \\
& \leq\|x-y\|^{2}-2 \lambda_{n} \beta\|B x-B y\|^{2}+\lambda_{n}^{2}\|B x-B y\|^{2} \\
& =\|x-y\|^{2}+\lambda_{n}\left(\lambda_{n}-2 \beta\right)\|B x-B y\|^{2} \\
& \leq\|x-y\|^{2} .
\end{aligned}
$$

It follows that $\left\|\left(I-\lambda_{n} B\right) x-\left(I-\lambda_{n} B\right) y\right\| \leq\|x-y\|$, hence $I-\lambda_{n} B$ is nonexpansive. 
Let $x^{*} \in \Theta, T_{r_{n}}$ be a sequence of mapping defined as in Lemma 2.1 and $u_{n}=T_{r_{n}} x_{n}$, for all $n \geq 0$, we have

$$
\left\|u_{n}-x^{*}\right\|=\left\|T_{r_{n}} x_{n}-T_{r_{n}} x^{*}\right\| \leq\left\|x_{n}-x^{*}\right\|
$$

By the fact that $P_{C}$ and $I-\lambda_{n} B$ are nonexpansive and $x^{*}=P_{C}\left(x^{*}-\lambda_{n} B x^{*}\right)$, we get

$$
\begin{aligned}
\left\|y_{n}-x^{*}\right\| & =\left\|\delta_{n} u_{n}+\left(1-\delta_{n}\right) P_{C}\left(u_{n}-\lambda_{n} B u_{n}\right)-x^{*}\right\| \\
& \leq \delta_{n}\left\|u_{n}-x^{*}\right\|+\left(1-\delta_{n}\right)\left\|P_{C}\left(u_{n}-\lambda_{n} B u_{n}\right)-P_{C}\left(x^{*}-\lambda_{n} B x^{*}\right)\right\| \\
& \leq \delta_{n}\left\|u_{n}-x^{*}\right\|+\left(1-\delta_{n}\right)\left\|\left(I-\lambda_{n} B\right) u_{n}-\left(I-\lambda_{n} B\right) x^{*}\right\| \\
& \leq \delta_{n}\left\|u_{n}-x^{*}\right\|+\left(1-\delta_{n}\right)\left\|u_{n}-x^{*}\right\| \\
& =\left\|u_{n}-x^{*}\right\| \\
& \leq\left\|x_{n}-x^{*}\right\| .
\end{aligned}
$$

Let $T_{n}=(1 /(n+1)) \sum_{i=0}^{n} T^{i} ;$ it follows that

$$
\begin{aligned}
\left\|T_{n} x-T_{n} y\right\| & =\left\|\frac{1}{n+1} \sum_{i=0}^{n} T^{i} x-\frac{1}{n+1} \sum_{i=0}^{n} T^{i} y\right\| \\
& \leq \frac{1}{n+1} \sum_{i=0}^{n}\left\|T^{i} x-T^{i} y\right\| \\
& \leq \frac{1}{n+1} \sum_{i=0}^{n}\|x-y\| \\
& =\frac{n+1}{n+1}\|x-y\| \\
& =\|x-y\|,
\end{aligned}
$$

which implies that $T_{n}$ is nonexpansive. Since $x^{*} \in \Theta$, we have $T_{n} x^{*}=(1 /(n+1)) \sum_{i=0}^{n} T^{i} x^{*}=$ $(1 /(n+1)) \sum_{i=0}^{n} x^{*}=x^{*}$, for all $x, y \in C$. By (3.5) and (3.6), we have

$$
\begin{aligned}
\left\|x_{n+1}-x^{*}\right\| & =\left\|\alpha_{n}\left(\gamma f\left(x_{n}\right)-A x^{*}\right)+\beta_{n}\left(x_{n}-x^{*}\right)+\left(\left(1-\beta_{n}\right) I-\alpha_{n} A\right)\left(T_{n} y_{n}-x^{*}\right)\right\| \\
& \leq \alpha_{n}\left\|\gamma f\left(x_{n}\right)-A x^{*}\right\|+\beta_{n}\left\|x_{n}-x^{*}\right\|+\left(1-\beta_{n}-\alpha_{n} \bar{\gamma}\right)\left\|y_{n}-x^{*}\right\|
\end{aligned}
$$


Fixed Point Theory and Applications

$$
\begin{aligned}
& \leq \alpha_{n}\left\|\gamma f\left(x_{n}\right)-A x^{*}\right\|+\beta_{n}\left\|x_{n}-x^{*}\right\|+\left(1-\beta_{n}-\alpha_{n} \bar{\gamma}\right)\left\|x_{n}-x^{*}\right\| \\
& \leq \alpha_{n} \gamma\left\|f\left(x_{n}\right)-f\left(x^{*}\right)\right\|+\alpha_{n}\left\|\gamma f\left(x^{*}\right)-A x^{*}\right\|+\left(1-\alpha_{n} \bar{\gamma}\right)\left\|x_{n}-x^{*}\right\| \\
& \leq \alpha_{n} \gamma \alpha\left\|x_{n}-x^{*}\right\|+\alpha_{n}\left\|\gamma f\left(x^{*}\right)-A x^{*}\right\|+\left(1-\alpha_{n} \bar{\gamma}\right)\left\|x_{n}-x^{*}\right\| \\
& =\left(1-\alpha_{n}(\bar{\gamma}-\gamma \alpha)\right)\left\|x_{n}-x^{*}\right\|+\alpha_{n}(\bar{\gamma}-\gamma \alpha) \frac{\left\|\gamma f\left(x^{*}\right)-A x^{*}\right\|}{(\bar{\gamma}-\gamma \alpha)} \\
& \leq \max \left\{\left\|x_{0}-x^{*}\right\|, \frac{\left\|\gamma f\left(x^{*}\right)-A x^{*}\right\|}{(\bar{\gamma}-\gamma \alpha)}\right\} .
\end{aligned}
$$

Hence $\left\{x_{n}\right\}$ is bounded and also $\left\{u_{n}\right\},\left\{y_{n}\right\}$ and $\left\{T_{n} y_{n}\right\}$ are bounded.

Next, we show that $\lim _{n \rightarrow \infty}\left\|x_{n+1}-x_{n}\right\|=0$. Observing that $u_{n}=T_{r_{n}} x_{n} \in \operatorname{dom} \varphi$ and $u_{n+1}=T_{r_{n+1}} x_{n+1} \in \operatorname{dom} \varphi$, we get

$$
\begin{gathered}
\phi\left(u_{n}, y\right)+\varphi(y)-\varphi\left(u_{n}\right)+\frac{1}{r_{n}}\left\langle y-u_{n}, u_{n}-x_{n}\right\rangle \geq 0, \quad \forall y \in C, \\
\phi\left(u_{n+1}, y\right)+\varphi(y)-\varphi\left(u_{n+1}\right)+\frac{1}{r_{n+1}}\left\langle y-u_{n+1}, u_{n+1}-x_{n+1}\right\rangle \geq 0, \quad \forall y \in C .
\end{gathered}
$$

Take $y=u_{n+1}$ in (3.8) and $y=u_{n}$ in (3.9), by using condition (A2); it follows that

$$
\left\langle u_{n+1}-u_{n}, \frac{u_{n}-x_{n}}{r_{n}}-\frac{u_{n+1}-x_{n+1}}{r_{n+1}}\right\rangle \geq 0
$$

Thus $\left\langle u_{n+1}-u_{n}, u_{n}-u_{n+1}+x_{n+1}-x_{n}+\left(1-r_{n} / r_{n+1}\right)\left(u_{n+1}-x_{n+1}\right)\right\rangle \geq 0$. Without loss of generality, let us assume that there exists a nonnegative real number $c$ such that $r_{n}>c$, for all $n \geq 1$. Then, we have

$$
\left\|u_{n+1}-u_{n}\right\|^{2} \leq\left\|u_{n+1}-u_{n}\right\|\left\{\left\|x_{n+1}-x_{n}\right\|+\left|1-\frac{r_{n}}{r_{n+1}}\right|\left\|u_{n+1}-x_{n+1}\right\|\right\}
$$

and hence

$$
\begin{aligned}
\left\|u_{n+1}-u_{n}\right\| & \leq\left\|x_{n+1}-x_{n}\right\|+\frac{1}{r_{n+1}}\left|r_{n+1}-r_{n}\right|\left\|u_{n+1}-x_{n+1}\right\| \\
& \leq\left\|x_{n+1}-x_{n}\right\|+\frac{1}{c}\left|r_{n+1}-r_{n}\right| M_{1}
\end{aligned}
$$


where $M_{1}=\sup \left\{\left\|u_{n}-x_{n}\right\|: n \in \mathbb{N}\right\}$. On the other hand, let $v_{n}=P_{C}\left(u_{n}-\lambda_{n} B u_{n}\right)$; it follows from the definition of $\left\{y_{n}\right\}$ that

$$
\begin{aligned}
\left\|y_{n+1}-y_{n}\right\|= & \|\left\{\delta_{n+1} u_{n+1}+\left(1-\delta_{n+1}\right) P_{C}\left(u_{n+1}-\lambda_{n+1} B u_{n+1}\right)\right\} \\
& -\left\{\delta_{n} u_{n}+\left(1-\delta_{n}\right) P_{C}\left(u_{n}-\lambda_{n} B u_{n}\right)\right\} \| \\
= & \| \delta_{n+1}\left(u_{n+1}-u_{n}\right)+\left(\delta_{n+1}-\delta_{n}\right) u_{n}+\left(1-\delta_{n+1}\right) P_{C}\left(u_{n+1}-\lambda_{n+1} B u_{n+1}\right) \\
& \quad-\left(1-\delta_{n+1}\right) P_{C}\left(u_{n}-\lambda_{n} B u_{n}\right)+\left(1-\delta_{n+1}\right) P_{C}\left(u_{n}-\lambda_{n} B u_{n}\right) \\
& \quad-\left(1-\delta_{n}\right) P_{C}\left(u_{n}-\lambda_{n} B u_{n}\right) \| \\
= & \| \delta_{n+1}\left(u_{n+1}-u_{n}\right)+\left(\delta_{n+1}-\delta_{n}\right) u_{n} \\
& \quad+\left(1-\delta_{n+1}\right)\left\{P_{C}\left(u_{n+1}-\lambda_{n+1} B u_{n+1}\right)-P_{C}\left(u_{n}-\lambda_{n} B u_{n}\right)\right\} \\
& \quad+\left(\delta_{n}-\delta_{n+1}\right) P_{C}\left(u_{n}-\lambda_{n} B u_{n}\right) \| \\
\leq & \delta_{n+1}\left\|u_{n+1}-u_{n}\right\|+\left|\delta_{n+1}-\delta_{n}\right|\left(\left\|u_{n}\right\|+\left\|v_{n}\right\|\right) \\
& +\left(1-\delta_{n+1}\right)\left\|\left(u_{n+1}-\lambda_{n+1} B u_{n+1}\right)-\left(u_{n}-\lambda_{n} B u_{n}\right)\right\| \\
= & \delta_{n+1}\left\|u_{n+1}-u_{n}\right\|+\left|\delta_{n+1}-\delta_{n}\right|\left(\left\|u_{n}\right\|+\left\|v_{n}\right\|\right) \\
& +\left(1-\delta_{n+1}\right) \|\left(u_{n+1}-\lambda_{n+1} B u_{n+1}\right)-\left(u_{n}-\lambda_{n+1} B u_{n}\right) \\
& \quad+\left(u_{n}-\lambda_{n+1} B u_{n}\right)-\left(u_{n}-\lambda_{n} B u_{n}\right) \| \\
\leq & \delta_{n+1}\left\|u_{n+1}-u_{n}\right\|+\left|\delta_{n+1}-\delta_{n}\right|\left(\left\|u_{n}\right\|+\left\|v_{n}\right\|\right) \\
& +\left(1-\delta_{n+1}\right)\left\{\left\|u_{n+1}-u_{n}\right\|+\left|\lambda_{n+1}-\lambda_{n}\right|\left\|B u_{n}\right\|\right\} \\
= & \delta_{n+1}-\delta_{n}\left|\left(\left\|u_{n}\right\|+\left\|v_{n}\right\|\right)+\left\|u_{n+1}-u_{n}\right\|+\left(1-\delta_{n+1}\right)\right| \lambda_{n+1}-\lambda_{n} \mid\left\|B u_{n}\right\| \\
\leq \mid & \delta_{n+1}-\delta_{n}\left|\left(\left\|u_{n}\right\|+\left\|v_{n}\right\|\right)+\left\|u_{n+1}-u_{n}\right\|+\right| \lambda_{n+1}-\lambda_{n} \mid\left\|B u_{n}\right\| \\
\leq \mid & \delta_{n+1}-\delta_{n}\left|\left(\left\|u_{n}\right\|+\left\|v_{n}\right\|\right)+\left\|x_{n+1}-x_{n}\right\|+\frac{1}{c}\right| r_{n+1}-r_{n} \mid M_{1} \\
& +\left|\lambda_{n+1}-\lambda_{n}\right|\left\|B u_{n}\right\| . \\
&
\end{aligned}
$$

We compute that

$$
\begin{aligned}
\left\|T_{n+1} y_{n+1}-T_{n} y_{n}\right\| & \leq\left\|T_{n+1} y_{n+1}-T_{n+1} y_{n}\right\|+\left\|T_{n+1} y_{n}-T_{n} y_{n}\right\| \\
& \leq\left\|y_{n+1}-y_{n}\right\|+\left\|\frac{1}{n+2} \sum_{i=0}^{n+1} T^{i} y_{n}-\frac{1}{n+1} \sum_{i=0}^{n} T^{i} y_{n}\right\| \\
& =\left\|y_{n+1}-y_{n}\right\|+\left\|\frac{1}{n+2} \sum_{i=0}^{n} T^{i} y_{n}+\frac{1}{n+2} T^{n+1} y_{n}-\frac{1}{n+1} \sum_{i=0}^{n} T^{i} y_{n}\right\|
\end{aligned}
$$


Fixed Point Theory and Applications

$$
\begin{aligned}
= & \left\|y_{n+1}-y_{n}\right\|+\left\|-\frac{1}{(n+1)(n+2)} \sum_{i=0}^{n} T^{i} y_{n}+\frac{1}{n+2} T^{n+1} y_{n}\right\| \\
\leq & \left\|y_{n+1}-y_{n}\right\|+\frac{1}{(n+1)(n+2)} \sum_{i=0}^{n}\left\|T^{i} y_{n}\right\|+\frac{1}{n+2}\left\|T^{n+1} y_{n}\right\| \\
\leq & \left\|y_{n+1}-y_{n}\right\|+\frac{1}{(n+1)(n+2)} \sum_{i=0}^{n}\left(\left\|T^{i} y_{n}-T^{i} x^{*}\right\|+\left\|x^{*}\right\|\right) \\
& +\frac{1}{n+2}\left(\left\|T^{n+1} y_{n}-T^{n+1} x^{*}\right\|+\left\|x^{*}\right\|\right) \\
\leq & \left\|y_{n+1}-y_{n}\right\|+\frac{1}{(n+1)(n+2)} \sum_{i=0}^{n}\left(\left\|y_{n}-x^{*}\right\|+\left\|x^{*}\right\|\right) \\
& +\frac{1}{n+2}\left(\left\|y_{n}-x^{*}\right\|+\left\|x^{*}\right\|\right) \\
\leq & \left\|y_{n+1}-y_{n}\right\|+\frac{n+1}{(n+1)(n+2)}\left(\left\|y_{n}-x^{*}\right\|+\left\|x^{*}\right\|\right) \\
& +\frac{1}{n+2}\left\|y_{n}-x^{*}\right\|+\frac{1}{n+2}\left\|x^{*}\right\| \\
= & \left\|y_{n+1}-y_{n}\right\|+\frac{2}{n+2}\left\|y_{n}-x^{*}\right\|+\frac{2}{n+2}\left\|x^{*}\right\| \\
\leq & \left|\delta_{n+1}-\delta_{n}\right|\left(\left\|u_{n}\right\|+\left\|v_{n}\right\|\right)+\left\|x_{n+1}-x_{n}\right\|+\frac{1}{c}\left|r_{n+1}-r_{n}\right| M_{1} \\
& +\left|\lambda_{n+1}-\lambda_{n}\right|\left\|B u_{n}\right\|+\frac{2}{n+2}\left\|y_{n}-x^{*}\right\|+\frac{2}{n+2}\left\|x^{*}\right\| .
\end{aligned}
$$

Let $x_{n+1}=\left(1-\beta_{n}\right) z_{n}+\beta_{n} x_{n}$; it follows that

$$
\begin{aligned}
z_{n} & =\frac{x_{n+1}-\beta_{n} x_{n}}{1-\beta_{n}} \\
& =\frac{\alpha_{n} \gamma f\left(x_{n}\right)+\left(\left(1-\beta_{n}\right) I-\alpha_{n} A\right) T_{n} y_{n}}{1-\beta_{n}},
\end{aligned}
$$

and hence

$$
\begin{aligned}
\left\|z_{n+1}-z_{n}\right\|= & \| \frac{\alpha_{n+1} \gamma f\left(x_{n+1}\right)+\left(\left(1-\beta_{n+1}\right) I-\alpha_{n+1} A\right) T_{n+1} y_{n+1}}{1-\beta_{n+1}} \\
& -\frac{\alpha_{n} \gamma f\left(x_{n}\right)+\left(\left(1-\beta_{n}\right) I-\alpha_{n} A\right) T_{n} y_{n}}{1-\beta_{n}} \|
\end{aligned}
$$




$$
\begin{aligned}
=\| & \frac{\alpha_{n+1} \gamma f\left(x_{n+1}\right)}{1-\beta_{n+1}}+\frac{\left(1-\beta_{n+1}\right) T_{n+1} y_{n+1}}{1-\beta_{n+1}} \\
& -\frac{\alpha_{n+1} A T_{n+1} y_{n+1}}{1-\beta_{n+1}}-\frac{\alpha_{n} \gamma f\left(x_{n}\right)}{1-\beta_{n}} \\
& \quad-\frac{\left(1-\beta_{n}\right) T_{n} y_{n}}{1-\beta_{n}}+\frac{\alpha_{n} A T_{n} y_{n}}{1-\beta_{n}} \| \\
= & \left\|\frac{\alpha_{n+1}}{1-\beta_{n+1}}\left(\gamma f\left(x_{n+1}\right)-A T_{n+1} y_{n+1}\right)+\frac{\alpha_{n}}{1-\beta_{n}}\left(A T_{n} y_{n}-\gamma f\left(x_{n}\right)\right)+T_{n+1} y_{n+1}-T_{n} y_{n}\right\| \\
\leq & \frac{\alpha_{n+1}}{1-\beta_{n+1}}\left\|\gamma f\left(x_{n+1}\right)-A T_{n+1} y_{n+1}\right\|+\frac{\alpha_{n}}{1-\beta_{n}}\left\|A T_{n} y_{n}-\gamma f\left(x_{n}\right)\right\|+\left\|T_{n+1} y_{n+1}-T_{n} y_{n}\right\| \\
\leq & \frac{\alpha_{n+1}}{1-\beta_{n+1}}\left\|\gamma f\left(x_{n+1}\right)-A T_{n+1} y_{n+1}\right\|+\frac{\alpha_{n}}{1-\beta_{n}}\left\|A T_{n} y_{n}-\gamma f\left(x_{n}\right)\right\| \\
& +\left|\delta_{n+1}-\delta_{n}\right|\left(\left\|u_{n}\right\|+\left\|v_{n}\right\|\right)+\left\|x_{n+1}-x_{n}\right\|+\frac{1}{c}\left|r_{n+1}-r_{n}\right| M_{1} \\
& +\left|\lambda_{n+1}-\lambda_{n}\right|\left\|B u_{n}\right\|+\frac{2}{n+2}\left\|y_{n}-x^{*}\right\|+\frac{2}{n+2}\left\|x^{*}\right\| .
\end{aligned}
$$

Therefore,

$$
\begin{aligned}
\left\|z_{n+1}-z_{n}\right\|-\left\|x_{n+1}-x_{n}\right\| \leq & \frac{\alpha_{n+1}}{1-\beta_{n+1}}\left\|\gamma f\left(x_{n+1}\right)-A T_{n+1} y_{n+1}\right\|+\frac{\alpha_{n}}{1-\beta_{n}}\left\|A T_{n} y_{n}-\gamma f\left(x_{n}\right)\right\| \\
& +\left|\delta_{n+1}-\delta_{n}\right|\left(\left\|u_{n}\right\|+\left\|v_{n}\right\|\right)+\frac{1}{c}\left\|r_{n+1}-r_{n}\right\| M_{1} \\
& +\left|\lambda_{n+1}-\lambda_{n}\right|\left\|B u_{n}\right\|+\frac{2}{n+2}\left\|y_{n}-x^{*}\right\|+\frac{2}{n+2}\left\|x^{*}\right\| .
\end{aligned}
$$

It follows from $n \rightarrow \infty$ and the conditions (i) $-(\mathrm{v})$, that

$$
\limsup _{n \rightarrow \infty}\left(\left\|z_{n+1}-z_{n}\right\|-\left\|x_{n+1}-x_{n}\right\|\right) \leq 0 .
$$

From Lemma 2.4 and (3.18), we obtain $\lim _{n \rightarrow \infty}\left\|z_{n}-x_{n}\right\|=0$ and also

$$
\lim _{n \rightarrow \infty}\left\|x_{n+1}-x_{n}\right\|=\lim _{n \rightarrow \infty}\left(1-\beta_{n}\right)\left\|z_{n}-x_{n}\right\|=0 .
$$


Fixed Point Theory and Applications

Next, we show that $\left\|x_{n}-u_{n}\right\| \rightarrow 0$ as $n \rightarrow \infty$. For $x^{*} \in \Theta$, we obtain

$$
\begin{aligned}
\left\|u_{n}-x^{*}\right\|^{2} & =\left\|T_{r_{n}} x_{n}-T_{r_{n}} x^{*}\right\|^{2} \\
& \leq\left\langle T_{r_{n}} x_{n}-T_{r_{n}} x^{*}, x_{n}-x^{*}\right\rangle \\
& =\left\langle u_{n}-x^{*}, x_{n}-x^{*}\right\rangle \\
& =\frac{1}{2}\left(\left\|u_{n}-x^{*}\right\|^{2}+\left\|x_{n}-x^{*}\right\|^{2}-\left\|u_{n}-x^{*}-x_{n}+x^{*}\right\|^{2}\right) \\
& =\frac{1}{2}\left(\left\|u_{n}-x^{*}\right\|^{2}+\left\|x_{n}-x^{*}\right\|^{2}-\left\|u_{n}-x_{n}\right\|^{2}\right),
\end{aligned}
$$

and hence

$$
\left\|u_{n}-x^{*}\right\|^{2} \leq\left\|x_{n}-x^{*}\right\|^{2}-\left\|u_{n}-x_{n}\right\|^{2}
$$

Since $\left\|y_{n}-x^{*}\right\| \leq\left\|u_{n}-x^{*}\right\|$ and from Lemma 2.3 and (3.21), we obtain

$$
\begin{aligned}
\left\|x_{n+1}-x^{*}\right\|^{2} & =\left\|\alpha_{n} \gamma f\left(x_{n}\right)+\beta_{n} x_{n}+\left(\left(1-\beta_{n}\right) I-\alpha_{n} A\right) T_{n} y_{n}-x^{*}\right\|^{2} \\
& =\left\|\alpha_{n}\left(\gamma f\left(x_{n}\right)-A x^{*}\right)+\beta_{n}\left(x_{n}-x^{*}\right)+\left(\left(1-\beta_{n}\right) I-\alpha_{n} A\right)\left(T_{n} y_{n}-x^{*}\right)\right\|^{2} \\
& \leq \alpha_{n}\left\|\gamma f\left(x_{n}\right)-A x^{*}\right\|^{2}+\beta_{n}\left\|x_{n}-x^{*}\right\|^{2}+\left(1-\beta_{n}-\alpha_{n} \bar{\gamma}\right)\left\|y_{n}-x^{*}\right\|^{2} \\
& \leq \alpha_{n}\left\|\gamma f\left(x_{n}\right)-A x^{*}\right\|^{2}+\beta_{n}\left\|x_{n}-x^{*}\right\|^{2}+\left(1-\beta_{n}-\alpha_{n} \bar{\gamma}\right)\left\|u_{n}-x^{*}\right\|^{2} \\
& \leq \alpha_{n}\left\|\gamma f\left(x_{n}\right)-A x^{*}\right\|^{2}+\beta_{n}\left\|x_{n}-x^{*}\right\|^{2}+\left(1-\beta_{n}-\alpha_{n} \bar{\gamma}\right)\left(\left\|x_{n}-x^{*}\right\|^{2}-\left\|x_{n}-u_{n}\right\|^{2}\right) \\
& \leq \alpha_{n}\left\|\gamma f\left(x_{n}\right)-A x^{*}\right\|^{2}+\left\|x_{n}-x^{*}\right\|^{2}-\left(1-\beta_{n}-\alpha_{n} \bar{\gamma}\right)\left\|x_{n}-u_{n}\right\|^{2} .
\end{aligned}
$$

Then, we have

$$
\begin{aligned}
\left(1-\beta_{n}-\alpha_{n} \bar{\gamma}\right)\left\|x_{n}-u_{n}\right\|^{2} & \leq \alpha_{n}\left\|\gamma f\left(x_{n}\right)-A x^{*}\right\|^{2}+\left\|x_{n}-x^{*}\right\|^{2}-\left\|x_{n+1}-x^{*}\right\|^{2} \\
& =\alpha_{n}\left\|\gamma f\left(x_{n}\right)-A x^{*}\right\|^{2}+\left\|x_{n}-x_{n+1}\right\|\left(\left\|x_{n}-x^{*}\right\|+\left\|x_{n+1}-x^{*}\right\|\right)
\end{aligned}
$$

By $\lim _{n \rightarrow \infty}\left\|x_{n+1}-x_{n}\right\|=0$, (i) and (iv), imply that

$$
\lim _{n \rightarrow \infty}\left\|u_{n}-x_{n}\right\|=0
$$

Since $\lim \inf _{n \rightarrow \infty} r_{n}>0$, we obtain

$$
\lim _{n \rightarrow \infty}\left\|\frac{x_{n}-u_{n}}{r_{n}}\right\|=\lim _{n \rightarrow \infty} \frac{1}{r_{n}}\left\|x_{n}-u_{n}\right\|=0 .
$$


Next, we show that $\lim _{n \rightarrow \infty}\left\|T_{n} y_{n}-x_{n}\right\|=0$. Indeed, observe that

$$
\begin{aligned}
\left\|x_{n}-T_{n} y_{n}\right\| \leq\left\|x_{n}-x_{n+1}\right\|+\left\|x_{n+1}-T_{n} y_{n}\right\| \\
=\left\|x_{n}-x_{n+1}\right\|+\left\|\alpha_{n} \gamma f\left(x_{n}\right)+\beta_{n} x_{n}+\left(\left(1-\beta_{n}\right) I-\alpha_{n} A\right) T_{n} y_{n}-T_{n} y_{n}\right\| \\
=\left\|x_{n}-x_{n+1}\right\|+\| \alpha_{n} \gamma f\left(x_{n}\right)-\alpha_{n} A T_{n} y_{n}+\alpha_{n} A T_{n} y_{n}+\beta_{n} x_{n}-\beta_{n} T_{n} y_{n}+\beta_{n} T_{n} y_{n} \\
\quad+\left(\left(1-\beta_{n}\right) I-\alpha_{n} A\right) T_{n} y_{n}-T_{n} y_{n} \| \\
\leq\left\|x_{n}-x_{n+1}\right\|+\alpha_{n}\left\|\gamma f\left(x_{n}\right)-A T_{n} y_{n}\right\|+\beta_{n}\left\|x_{n}-T_{n} y_{n}\right\|
\end{aligned}
$$

and then

$$
\left\|x_{n}-T_{n} y_{n}\right\| \leq \frac{1}{1-\beta_{n}}\left\|x_{n}-x_{n+1}\right\|+\frac{\alpha_{n}}{1-\beta_{n}}\left\|\gamma f\left(x_{n}\right)-A T_{n} y_{n}\right\|
$$

Since $\lim _{n \rightarrow \infty}\left\|x_{n+1}-x_{n}\right\|=0$, (i) and (iv), we get $\lim _{n \rightarrow \infty}\left\|x_{n}-T_{n} y_{n}\right\|=0$.

Next, we show that $\lim _{n \rightarrow \infty}\left\|y_{n}-v_{n}\right\|=0$, where $v_{n}=P_{C}\left(u_{n}-\lambda_{n} B u_{n}\right)$. From Lemma 2.3 and (3.3), we obtain

$$
\begin{aligned}
\left\|x_{n+1}-x^{*}\right\|^{2} \leq & \alpha_{n}\left\|\gamma f\left(x_{n}\right)-A x^{*}\right\|^{2}+\beta_{n}\left\|x_{n}-x^{*}\right\|^{2}+\left(\left(1-\beta_{n}\right) I-\alpha_{n} A\right)\left\|T_{n} y_{n}-x^{*}\right\|^{2} \\
\leq & \alpha_{n}\left\|\gamma f\left(x_{n}\right)-A x^{*}\right\|^{2}+\beta_{n}\left\|x_{n}-x^{*}\right\|^{2}+\left(1-\beta_{n}-\alpha_{n} \bar{\gamma}\right)\left\|y_{n}-x^{*}\right\|^{2} \\
= & \alpha_{n}\left\|\gamma f\left(x_{n}\right)-A x^{*}\right\|^{2}+\beta_{n}\left\|x_{n}-x^{*}\right\|^{2} \\
& +\left(1-\beta_{n}-\alpha_{n} \bar{\gamma}\right)\left\|\delta_{n}\left(u_{n}-x^{*}\right)+\left(1-\delta_{n}\right)\left\{P_{C}\left(u_{n}-\lambda_{n} B u_{n}\right)-P_{C}\left(x^{*}-\lambda_{n} B x^{*}\right)\right\}\right\|^{2} \\
\leq & \alpha_{n}\left\|\gamma f\left(x_{n}\right)-A x^{*}\right\|^{2}+\beta_{n}\left\|x_{n}-x^{*}\right\|^{2}+\left(1-\beta_{n}-\alpha_{n} \bar{\gamma}\right) \delta_{n}\left\|u_{n}-x^{*}\right\|^{2} \\
& +\left(1-\beta_{n}-\alpha_{n} \bar{\gamma}\right)\left(1-\delta_{n}\right)\left\|\left(u_{n}-\lambda_{n} B u_{n}\right)-\left(x^{*}-\lambda_{n} B x^{*}\right)\right\|^{2} \\
= & \alpha_{n}\left\|\gamma f\left(x_{n}\right)-A x^{*}\right\|^{2}+\beta_{n}\left\|x_{n}-x^{*}\right\|^{2}+\left(1-\beta_{n}-\alpha_{n} \bar{\gamma}\right) \delta_{n}\left\|u_{n}-x^{*}\right\|^{2} \\
& +\left(1-\beta_{n}-\alpha_{n} \bar{\gamma}\right)\left(1-\delta_{n}\right)\left\|\left(u_{n}-x^{*}\right)-\lambda_{n}\left(B u_{n}-B x^{*}\right)\right\|^{2} \\
\leq & \alpha_{n}\left\|\gamma f\left(x_{n}\right)-A x^{*}\right\|^{2}+\beta_{n}\left\|x_{n}-x^{*}\right\|^{2}+\left(1-\beta_{n}-\alpha_{n} \bar{\gamma}\right) \delta_{n}\left\|u_{n}-x^{*}\right\|^{2}
\end{aligned}
$$


Fixed Point Theory and Applications

$$
\begin{aligned}
& +\left(1-\beta_{n}-\alpha_{n} \bar{\gamma}\right)\left(1-\delta_{n}\right)\left\{\left\|x_{n}-x^{*}\right\|^{2}+\lambda_{n}\left(\lambda_{n}-2 \beta\right)\left\|B u_{n}-B x^{*}\right\|^{2}\right\} \\
\leq & \alpha_{n}\left\|\gamma f\left(x_{n}\right)-A x^{*}\right\|^{2}+\left(1-\alpha_{n} \bar{\gamma}\right)\left\|x_{n}-x^{*}\right\|^{2} \\
& +\left(1-\beta_{n}-\alpha_{n} \bar{\gamma}\right)\left(1-\delta_{n}\right) \lambda_{n}\left(\lambda_{n}-2 \beta\right)\left\|B u_{n}-B x^{*}\right\|^{2} \\
\leq & \alpha_{n}\left\|\gamma f\left(x_{n}\right)-A x^{*}\right\|^{2}+\left\|x_{n}-x^{*}\right\|^{2} \\
& +\left(1-\beta_{n}-\alpha_{n} \bar{\gamma}\right)\left(1-\delta_{n}\right) a(b-2 \beta)\left\|B u_{n}-B x^{*}\right\|^{2} .
\end{aligned}
$$

It follows that

$$
\begin{aligned}
0 & \leq\left(1-\beta_{n}-\alpha_{n} \bar{\gamma}\right)\left(1-\delta_{n}\right) a(2 \beta-b)\left\|B u_{n}-B x^{*}\right\|^{2} \\
& \leq \alpha_{n}\left\|\gamma f\left(x_{n}\right)-A x^{*}\right\|^{2}+\left\|x_{n}-x^{*}\right\|^{2}-\left\|x_{n+1}-x^{*}\right\|^{2} \\
& \leq \alpha_{n}\left\|\gamma f\left(x_{n}\right)-A x^{*}\right\|^{2}+\left\|x_{n+1}-x_{n}\right\|\left(\left\|x_{n}-x^{*}\right\|+\left\|x_{n+1}-x^{*}\right\|\right) .
\end{aligned}
$$

Since $\alpha_{n} \rightarrow 0$ and $\left\|x_{n+1}-x_{n}\right\| \rightarrow 0$, as $n \rightarrow \infty$, we obtain $\left\|B u_{n}-B x^{*}\right\| \rightarrow 0$ as $n \rightarrow \infty$. Using (2.1), we have

$$
\begin{aligned}
\left\|v_{n}-x^{*}\right\|^{2}= & \left\|P_{C}\left(u_{n}-\lambda_{n} B u_{n}\right)-P_{C}\left(x^{*}-\lambda_{n} B x^{*}\right)\right\|^{2} \\
\leq & \left\langle\left(u_{n}-\lambda_{n} B u_{n}\right)-\left(x^{*}-\lambda_{n} B x^{*}\right), v_{n}-x^{*}\right\rangle \\
= & \frac{1}{2}\left\{\left\|\left(u_{n}-\lambda_{n} B u_{n}\right)-\left(x^{*}-\lambda_{n} B x^{*}\right)\right\|^{2}+\left\|v_{n}-x^{*}\right\|^{2}\right\} \\
& \quad-\frac{1}{2}\left\{\left\|\left(u_{n}-\lambda_{n} B u_{n}\right)-\left(x^{*}-\lambda_{n} B x^{*}\right)-\left(v_{n}-x^{*}\right)\right\|^{2}\right\} \\
= & \frac{1}{2}\left\{\left\|u_{n}-x^{*}\right\|^{2}+\left\|v_{n}-x^{*}\right\|^{2}-\left\|\left(u_{n}-v_{n}\right)-\lambda_{n}\left(B u_{n}-B x^{*}\right)\right\|^{2}\right\} \\
= & \frac{1}{2}\left\{\left\|u_{n}-x^{*}\right\|^{2}+\left\|v_{n}-x^{*}\right\|^{2}\right. \\
& \left.\quad-\left(\left\|u_{n}-v_{n}\right\|^{2}+\lambda_{n}^{2}\left\|B u_{n}-B x^{*}\right\|^{2}-2 \lambda_{n}\left\langle u_{n}-v_{n}, B u_{n}-B x^{*}\right\rangle\right)\right\} \\
\leq & \frac{1}{2}\left\{\left\|u_{n}-x^{*}\right\|^{2}+\left\|v_{n}-x^{*}\right\|^{2}-\left\|u_{n}-v_{n}\right\|^{2}-\lambda_{n}^{2}\left\|B u_{n}-B x^{*}\right\|^{2}\right. \\
& \left.\quad+2 \lambda_{n}\left\langle u_{n}-v_{n}, B u_{n}-B x^{*}\right\rangle\right\},
\end{aligned}
$$

so, we obtain

$$
\left\|v_{n}-x^{*}\right\|^{2} \leq\left\|u_{n}-x^{*}\right\|^{2}-\left\|u_{n}-v_{n}\right\|^{2}-\lambda_{n}^{2}\left\|B u_{n}-B x^{*}\right\|^{2}+2 \lambda_{n}\left\langle u_{n}-v_{n}, B u_{n}-B x^{*}\right\rangle,
$$


and hence

$$
\begin{aligned}
\left\|x_{n+1}-x^{*}\right\|^{2} \leq & \alpha_{n}\left\|\gamma f\left(x_{n}\right)-A x^{*}\right\|^{2}+\beta_{n}\left\|x_{n}-x^{*}\right\|^{2}+\left(1-\beta_{n}-\alpha_{n} \bar{\gamma}\right)\left\|y_{n}-x^{*}\right\|^{2} \\
= & \alpha_{n}\left\|\gamma f\left(x_{n}\right)-A x^{*}\right\|^{2}+\beta_{n}\left\|x_{n}-x^{*}\right\|^{2}+\left(1-\beta_{n}-\alpha_{n} \bar{\gamma}\right)\left\|\delta_{n} u_{n}+\left(1-\delta_{n}\right) v_{n}-x^{*}\right\|^{2} \\
\leq & \alpha_{n}\left\|\gamma f\left(x_{n}\right)-A x^{*}\right\|^{2}+\beta_{n}\left\|x_{n}-x^{*}\right\|^{2}+\left(1-\beta_{n}-\alpha_{n} \bar{\gamma}\right) \delta_{n}\left\|u_{n}-x^{*}\right\|^{2} \\
& +\left(1-\beta_{n}-\alpha_{n} \bar{\gamma}\right)\left(1-\delta_{n}\right)\left\|v_{n}-x^{*}\right\|^{2} \\
\leq & \alpha_{n}\left\|\gamma f\left(x_{n}\right)-A x^{*}\right\|^{2}+\beta_{n}\left\|x_{n}-x^{*}\right\|^{2}+\left(1-\beta_{n}-\alpha_{n} \bar{\gamma}\right) \delta_{n}\left\|u_{n}-x^{*}\right\|^{2} \\
& +\left(1-\beta_{n}-\alpha_{n} \bar{\gamma}\right)\left\|v_{n}-x^{*}\right\|^{2} \\
\leq & \alpha_{n}\left\|\gamma f\left(x_{n}\right)-A x^{*}\right\|^{2}+\beta_{n}\left\|x_{n}-x^{*}\right\|^{2}+\left(1-\beta_{n}-\alpha_{n} \bar{\gamma}\right) \delta_{n}\left\|u_{n}-x^{*}\right\|^{2} \\
& +\left(1-\beta_{n}-\alpha_{n} \bar{\gamma}\right)\left\{\left\|u_{n}-x^{*}\right\|^{2}-\left\|u_{n}-v_{n}\right\|^{2}-\lambda_{n}^{2}\left\|B u_{n}-B x^{*}\right\|^{2}\right. \\
& \left.\quad+2 \lambda_{n}\left\langle u_{n}-v_{n}, B u_{n}-B x^{*}\right\rangle\right\} \\
& -\left(1-\beta_{n}-\alpha_{n} \bar{\gamma}\right) \lambda_{n}^{2}\left\|B u_{n}-B x^{*}\right\|^{2}+\left(1-\beta_{n}-\alpha_{n} \bar{\gamma}\right) 2 \lambda_{n}\left\|u_{n}-v_{n}\right\|\left\|B u_{n}-B x^{*}\right\|, \\
\leq & \alpha_{n}\left\|\gamma f\left(x_{n}\right)-A x^{*}\right\|^{2}+\beta_{n}\left\|x_{n}-x^{*}\right\|^{2}+\left(1-\beta_{n}-\alpha_{n} \bar{\gamma}\right) \delta_{n}\left\|u_{n}-x^{*}\right\|^{2} \\
& +\left(1-\beta_{n}-\alpha_{n} \bar{\gamma}\right)\left\|x_{n}-x^{*}\right\|^{2}-\left(1-\beta_{n}-\alpha_{n} \bar{\gamma}\right)\left\|u_{n}-v_{n}\right\|^{2} \\
& -\left(1-\beta_{n}-\alpha_{n} \bar{\gamma}\right) \lambda_{n}^{2}\left\|B u_{n}-B x^{*}\right\|^{2}+\left(1-\beta_{n}-\alpha_{n} \bar{\gamma}\right) 2 \lambda_{n}\left\langle u_{n}-v_{n}, B u_{n}-B x^{*}\right\rangle \\
& \alpha_{n}\left\|\gamma f\left(x_{n}\right)-A x^{*}\right\|^{2}+\left\|x_{n}-x^{*}\right\|^{2}+\left(1-\beta_{n}-\alpha_{n} \bar{\gamma}\right) \delta_{n}\left\|u_{n}-x^{*}\right\|^{2} \\
& -\left(1-\beta_{n}-\alpha_{n} \bar{\gamma}\right)\left\|u_{n}-v_{n}\right\|^{2} \\
& \\
& \\
& \\
&
\end{aligned}
$$

which implies that

$$
\begin{aligned}
\left(1-\beta_{n}-\alpha_{n} \bar{\gamma}\right)\left\|u_{n}-v_{n}\right\|^{2} \leq & \alpha_{n}\left\|\gamma f\left(x_{n}\right)-A x^{*}\right\|^{2}+\left\|x_{n}-x^{*}\right\|^{2}-\left\|x_{n+1}-x^{*}\right\|^{2} \\
& +\left(1-\beta_{n}-\alpha_{n} \bar{\gamma}\right) \delta_{n}\left\|u_{n}-x^{*}\right\|^{2} \\
& -\left(1-\beta_{n}-\alpha_{n} \bar{\gamma}\right) \lambda_{n}^{2}\left\|B u_{n}-B x^{*}\right\|^{2} \\
& +\left(1-\beta_{n}-\alpha_{n} \bar{\gamma}\right) 2 \lambda_{n}\left\|u_{n}-v_{n}\right\|\left\|B u_{n}-B x^{*}\right\|
\end{aligned}
$$


Fixed Point Theory and Applications

$$
\begin{aligned}
\leq & \alpha_{n}\left\|\gamma \mathrm{f}\left(x_{n}\right)-A x^{*}\right\|^{2}+\left\|x_{n}-x_{n+1}\right\|\left(\left\|x_{n}-x^{*}\right\|+\left\|x_{n+1}-x^{*}\right\|\right) \\
& +\left(1-\beta_{n}-\alpha_{n} \bar{\gamma}\right) \delta_{n}\left\|u_{n}-x^{*}\right\|^{2}-\left(1-\beta_{n}-\alpha_{n} \bar{\gamma}\right) \lambda_{n}^{2}\left\|B u_{n}-B x^{*}\right\|^{2} \\
& +\left(1-\beta_{n}-\alpha_{n} \bar{\gamma}\right) 2 \lambda_{n}\left\|u_{n}-v_{n}\right\|\left\|B u_{n}-B x^{*}\right\| .
\end{aligned}
$$

Since $\left\|B u_{n}-B x^{*}\right\| \rightarrow 0,\left\|x_{n+1}-x_{n}\right\| \rightarrow 0$ as $n \rightarrow 0$ and the condition (i)-(iii), we have $\left\|u_{n}-v_{n}\right\| \rightarrow 0$ as $n \rightarrow 0$. At the same time, we note that

$$
\left\|y_{n}-v_{n}\right\|=\left\|\delta_{n}\left(u_{n}-v_{n}\right)\right\|=\delta_{n}\left\|u_{n}-v_{n}\right\|
$$

since $\delta_{n} \rightarrow 0$, we have $\left\|y_{n}-v_{n}\right\| \rightarrow 0$ as $n \rightarrow \infty$. Consequently, we observe that

$$
\left\|T_{n} y_{n}-y_{n}\right\| \leq\left\|T_{n} y_{n}-x_{n}\right\|+\left\|x_{n}-u_{n}\right\|+\left\|u_{n}-v_{n}\right\|+\left\|v_{n}-y_{n}\right\| \longrightarrow 0, \quad \text { as } n \longrightarrow \infty
$$

It is easy to see that $P_{\Theta}(I-A+\gamma f)(z)$ is a contradiction of $H$ into itself. Hence $H$ is complete, there exists a unique fixed point $z \in H$, such that $z=P_{\Theta}(I-A+\gamma f)(z)$.

Next, we show that

$$
\limsup _{n \rightarrow \infty}\left\langle(A-\gamma f) z, \mathrm{z}-x_{n}\right\rangle \leq 0
$$

Indeed, we can choose a subsequence $\left\{y_{n_{i}}\right\}$ of $\left\{y_{n}\right\}$, such that

$$
\lim _{i \rightarrow \infty}\left\langle(A-\gamma f) z, z-y_{n_{i}}\right\rangle=\limsup _{n \rightarrow \infty}\left\langle(A-\gamma f) z, z-y_{n}\right\rangle
$$

Since $\left\{y_{n_{i}}\right\}$ is bounded, there exists a subsequence $\left\{y_{n_{i j}}\right\}$ of $\left\{y_{n_{i}}\right\}$ which converge weakly to $\bar{y} \in C$. Without loss of generality, we can assume that $y_{n_{i}}-\bar{y}$. From $\left\|T_{n} y_{n}-y_{n}\right\| \rightarrow 0$, we obtain $T_{n} y_{n_{i}} \rightarrow \bar{y}$.

Let us show $\bar{y} \in \operatorname{MEP}(\phi, \varphi)$. Since $u_{n}=T_{r_{n}} x_{n} \in \operatorname{dom} \varphi$, we obtain

$$
\phi\left(u_{n}, y\right)+\varphi(y)-\varphi\left(u_{n}\right)+\frac{1}{r_{n}}\left\langle y-u_{n}, u_{n}-x_{n}\right\rangle \geq 0, \quad \forall y \in C
$$

From (A2), we also have

$$
\varphi(y)-\varphi\left(u_{n}\right)+\frac{1}{r_{n}}\left\langle y-u_{n}, u_{n}-x_{n}\right\rangle \geq \phi\left(y, u_{n}\right), \quad \forall y \in C
$$

and hence

$$
\varphi(y)-\varphi\left(u_{n}\right)+\left\langle y-u_{n_{i}}, \frac{u_{n_{i}}-x_{n_{i}}}{r_{n_{i}}}\right\rangle \geq \phi\left(y, u_{n_{i}}\right), \quad \forall y \in C
$$


From $\left\|u_{n}-x_{n}\right\| \rightarrow 0,\left\|x_{n}-T_{n} y_{n}\right\| \rightarrow 0$ and $\left\|T_{n} y_{n}-y_{n}\right\| \rightarrow 0$, we get $u_{n_{i}} \rightarrow \bar{y}$. Since $\left(u_{n_{i}}-\right.$ $\left.x_{n_{i}}\right) / r_{n_{i}} \rightarrow 0$, thus that from (A4) and the weakly lower semicontinuity of $\varphi$ that $\phi(y, \bar{y})+$ $\varphi(\bar{y})-\varphi(y) \leq 0$, for all $y \in C$. For $t$ with $0<t \leq 1$ and $x \in C$, let $x_{t}=t x+(1-t) \bar{y}$. Since $x \in C$ and $\bar{y} \in C$, we have $x_{t} \in C$ and hence $\phi\left(x_{t}, \bar{y}\right)+\varphi(\bar{y})-\varphi\left(x_{t}\right) \leq 0$. So, from (A1), (A4) and the convexity of $\varphi$, we have

$$
\begin{aligned}
0 & =\phi\left(x_{t}, x_{t}\right)+\varphi\left(x_{t}\right)-\varphi\left(x_{t}\right) \\
& \leq t \phi\left(x_{t}, x\right)+(1-t) \phi\left(x_{t}, \bar{y}\right)+t \varphi(x)+(1-t) \varphi(\bar{y})-\varphi\left(x_{t}\right) \\
& =t\left(\phi\left(x_{t}, x\right)+\varphi(x)-\varphi\left(x_{t}\right)\right)+(1-t)\left(\phi\left(x_{t}, \bar{y}\right)+\varphi(\bar{y})-\varphi\left(x_{t}\right)\right) \\
& \leq t\left(\phi\left(x_{t}, x\right)+\varphi(x)-\varphi\left(x_{t}\right)\right)
\end{aligned}
$$

Dividing by $t$, we get $\phi\left(x_{t}, x\right)+\varphi(x)-\varphi\left(x_{t}\right) \geq 0$. From (A3) and the weakly lower semicontinuity of $\varphi$, we have $\phi(\bar{y}, y)+\varphi(y)-\varphi(\bar{y}) \geq 0$, for all $y \in C$ and hence $\bar{y} \in \operatorname{MEP}(\phi, \varphi)$.

Next, we show that $\bar{y} \in F\left(T_{n}\right)=(1 /(n+1)) \sum_{i=0}^{n} F\left(T^{i}\right)$. Assume that $\bar{y} \notin(1 /(n+$ 1)) $\sum_{i=0}^{n} F\left(T^{i}\right)$, since $y_{n_{i}} \rightarrow \bar{y}$ and $T_{n} \bar{y} \neq \bar{y}$. From Opial's condition, we have

$$
\begin{aligned}
\liminf _{i \rightarrow \infty}\left\|y_{n_{i}}-\bar{y}\right\| & <\liminf _{i \rightarrow \infty}\left\|y_{n_{i}}-T_{n} \bar{y}\right\| \\
& \leq \liminf _{i \rightarrow \infty}\left(\left\|y_{n_{i}}-T_{n} y_{n_{i}}\right\|+\left\|T_{n} y_{n_{i}}-T_{n} \bar{y}\right\|\right) \\
& \leq \liminf _{i \rightarrow \infty}\left\|y_{n_{i}}-\bar{y}\right\|,
\end{aligned}
$$

which is a contradiction. Thus, we obtain $\bar{y} \in F\left(T_{n}\right)=(1 /(n+1)) \sum_{i=0}^{n} F\left(T^{i}\right)$.

Now, let us show that $v \in \operatorname{VI}(C, B)$. Let $U: H \rightarrow 2^{H}$ be a set-valued mapping is defined by

$$
U v= \begin{cases}B v+N_{C} v, & v \in C, \\ \emptyset, & v \notin C,\end{cases}
$$

where $N_{C} v$ is the normal cone to $C$ at $v \in C$. We have $U$ is maximal monotone and $0 \in U v$ if and only if $v \in \operatorname{VI}(C, B)$. Let $(v, w) \in G(U)$, hence $w-B v \in N_{C} v$ and $v_{n} \in C$, we have $\left\langle v-v_{n}, w-B v\right\rangle \geq 0$. On the other hand, from $v_{n}=P_{C}\left(u_{n}-\lambda_{n} B u_{n}\right)$, we have

$$
\left\langle v-v_{n}, v_{n}-\left(u_{n}-\lambda_{n} B u_{n}\right)\right\rangle \geq 0,
$$

that is

$$
\left\langle v-v_{n}, \frac{v_{n}-u_{n}}{\lambda_{n}}+B u_{n}\right\rangle \geq 0
$$


Therefor, we have

$$
\begin{aligned}
\left\langle v-v_{n_{i}}, w\right\rangle & \geq\left\langle v-v_{n_{i}}, B v\right\rangle \\
& \geq\left\langle v-v_{n_{i}}, B v\right\rangle-\left\langle v-v_{n_{i}}, \frac{v_{n_{i}}-u_{n_{i}}}{\lambda_{n_{i}}}+B u_{n_{i}}\right\rangle \\
& =\left\langle v-v_{n_{i}}, B v-\frac{v_{n_{i}}-u_{n_{i}}}{\lambda_{n_{i}}}-B u_{n_{i}}\right\rangle \\
& =\left\langle v-v_{n_{i}}, B v-B v_{n_{i}}\right\rangle+\left\langle v-v_{n_{i}}, B v_{n_{i}}-B u_{n_{i}}\right\rangle-\left\langle v-v_{n_{i}}, \frac{v_{n_{i}}-u_{n_{i}}}{\lambda_{n_{i}}}\right\rangle \\
& \geq\left\langle v-v_{n_{i}}, B v_{n_{i}}-B u_{n_{i}}\right\rangle-\left\langle v-v_{n_{i}}, \frac{v_{n_{i}}-u_{n_{i}}}{\lambda_{n_{i}}}\right\rangle \\
& \geq\left\|v-v_{n_{i}}\right\|\left\|B v_{n_{i}}-B u_{n_{i}}\right\|-\left\|v-v_{n_{i}}\right\|\left\|\frac{v_{n_{i}}-u_{n_{i}}}{\lambda_{n_{i}}}\right\| .
\end{aligned}
$$

Noting that $\left\|v_{n_{i}}-u_{n_{i}}\right\| \rightarrow 0$ as $i \rightarrow \infty$ and $B$ is $\beta$-inverse-strongly monotone, hence from (3.46), we obtain $\langle v-\bar{y}, w\rangle \geq 0$ as $i \rightarrow \infty$. Since $U$ is maximal monotone, we have $\bar{y} \in U^{-1} 0$, and hence $\bar{y} \in \operatorname{VI}(C, B)$. Therefore, $\bar{y} \in \Theta:=\bigcap_{i=1}^{n} F\left(T^{i}\right) \cap \operatorname{VI}(C, B) \cap \operatorname{MEP}(\phi, \varphi)$.

Since $z=P_{\Theta}(I-A+\gamma f)(z)$, we have

$$
\begin{aligned}
\limsup _{n \rightarrow \infty}\left\langle(\gamma f-A) z, x_{n}-z\right\rangle & =\limsup _{n \rightarrow \infty}\left\langle(\gamma f-A) z, T_{n} y_{n}-z\right\rangle \\
& =\limsup _{i \rightarrow \infty}\left\langle(\gamma f-A) z, T_{n} y_{n_{i}}-z\right\rangle \\
& =\langle(\gamma f-A) z, \bar{y}-z\rangle \leq 0 .
\end{aligned}
$$

Finally, we show that $\left\{x_{n}\right\}$ converge strongly to $z$, we obtain that

$$
\begin{aligned}
\left\|x_{n+1}-z\right\|^{2} & =\left\|\alpha_{n} \gamma f\left(x_{n}\right)+\beta_{n} x_{n}+\left(\left(1-\beta_{n}\right) I-\alpha_{n} A\right) T_{n} y_{n}-z\right\|^{2} \\
& =\left\|\alpha_{n}\left(\gamma f\left(x_{n}\right)-A z\right)+\beta_{n}\left(x_{n}-z\right)+\left(\left(1-\beta_{n}\right) I-\alpha_{n} A\right)\left(T_{n} y_{n}-z\right)\right\|^{2} \\
& =\alpha_{n}^{2}\left\|\gamma f\left(x_{n}\right)-A z\right\|^{2}+\left\|\beta_{n}\left(x_{n}-z\right)+\left(\left(1-\beta_{n}\right) I-\alpha_{n} A\right)\left(T_{n} y_{n}-z\right)\right\|^{2}
\end{aligned}
$$


Fixed Point Theory and Applications

$$
\begin{aligned}
& +2\left\langle\beta_{n}\left(x_{n}-z\right)+\left(\left(1-\beta_{n}\right) I-\alpha_{n} A\right)\left(T_{n} y_{n}-z\right), \alpha_{n}\left(\gamma f\left(x_{n}\right)-A z\right)\right\rangle \\
& \leq \alpha_{n}^{2}\left\|\gamma f\left(x_{n}\right)-A z\right\|^{2}+\left\{\beta_{n}\left\|x_{n}-z\right\|+\left(1-\beta_{n}-\alpha_{n} \bar{\gamma}\right)\left\|y_{n}-z\right\|\right\}^{2} \\
& +2 \alpha_{n} \beta_{n}\left\langle x_{n}-z, \gamma f\left(x_{n}\right)-A z\right\rangle+2 \alpha_{n}\left(1-\beta_{n}-\alpha_{n} \bar{\gamma}\right)\left\langle T_{n} y_{n}-z, \gamma f\left(x_{n}\right)-A z\right\rangle \\
& \leq \alpha_{n}^{2}\left\|\gamma f\left(x_{n}\right)-A z\right\|^{2}+\left\{\beta_{n}\left\|x_{n}-z\right\|+\left(1-\beta_{n}-\alpha_{n} \bar{\gamma}\right)\left\|x_{n}-z\right\|\right\}^{2} \\
& +2 \alpha_{n} \beta_{n}\left\langle x_{n}-z, \gamma f\left(x_{n}\right)-\gamma f(z)\right\rangle+2 \alpha_{n} \beta_{n}\left\langle x_{n}-z, \gamma f(z)-A z\right\rangle \\
& +2 \alpha_{n}\left(1-\beta_{n}-\alpha_{n} \bar{\gamma}\right)\left\langle T_{n} y_{n}-z, \gamma f\left(x_{n}\right)-\gamma f(z)\right\rangle \\
& +2 \alpha_{n}\left(1-\beta_{n}-\alpha_{n} \bar{\gamma}\right)\left\langle T_{n} y_{n}-z, \gamma f(z)-A z\right\rangle \\
& \leq \alpha_{n}^{2}\left\|\gamma f\left(x_{n}\right)-A z\right\|^{2}+\left(1-\alpha_{n} \bar{\gamma}\right)^{2}\left\|x_{n}-z\right\|^{2}+2 \alpha_{n} \beta_{n} \gamma\left\|x_{n}-z\right\|\left\|f\left(x_{n}\right)-f(z)\right\| \\
& +2 \alpha_{n} \beta_{n}\left\langle x_{n}-z, \gamma f(z)-A z\right\rangle+2 \alpha_{n}\left(1-\beta_{n}-\alpha_{n} \bar{\gamma}\right) \gamma\left\|T_{n} y_{n}-z\right\|\left\|f\left(x_{n}\right)-f(z)\right\| \\
& +2 \alpha_{n}\left(1-\beta_{n}-\alpha_{n} \bar{\gamma}\right)\left\langle T_{n} y_{n}-z, \gamma f(z)-A z\right\rangle \\
& \leq \alpha_{n}^{2}\left\|\gamma f\left(x_{n}\right)-A z\right\|^{2}+\left(1-\alpha_{n} \bar{\gamma}\right)^{2}\left\|x_{n}-z\right\|^{2}+2 \alpha_{n} \beta_{n} \gamma \alpha\left\|x_{n}-z\right\|^{2} \\
& +2 \alpha_{n} \beta_{n}\left\langle x_{n}-z, \gamma f(z)-A z\right\rangle+2 \alpha_{n}\left(1-\beta_{n}-\alpha_{n} \bar{\gamma}\right) \gamma \alpha\left\|x_{n}-z\right\|^{2} \\
& +2 \alpha_{n}\left(1-\beta_{n}-\alpha_{n} \bar{\gamma}\right)\left\langle T_{n} y_{n}-z, \gamma f(z)-A z\right\rangle \\
& =\alpha_{n}^{2}\left\|\gamma f\left(x_{n}\right)-A z\right\|^{2}+\left(1-2 \alpha_{n} \bar{\gamma}+\alpha_{n}^{2} \bar{\gamma}^{2}+2 \alpha_{n} \gamma \alpha-2 \alpha_{n}^{2} \bar{\gamma} \gamma \alpha\right)\left\|x_{n}-z\right\|^{2} \\
& +2 \alpha_{n} \beta_{n}\left\langle x_{n}-z, \gamma f(z)-A z\right\rangle+2 \alpha_{n}\left(1-\beta_{n}-\alpha_{n} \bar{\gamma}\right)\left\langle T_{n} y_{n}-z, \gamma f(z)-A z\right\rangle \\
& \leq\left\{1-\alpha_{n}\left(2 \bar{\gamma}-\alpha_{n} \bar{\gamma}^{2}-2 \gamma \alpha+2 \alpha_{n} \bar{\gamma} \gamma \alpha\right)\right\}\left\|x_{n}-z\right\|^{2}+\alpha_{n}^{2}\left\|\gamma f\left(x_{n}\right)-A z\right\|^{2} \\
& +2 \alpha_{n} \beta_{n}\left\langle x_{n}-z, \gamma f(z)-A z\right\rangle+2 \alpha_{n}\left(1-\beta_{n}-\alpha_{n} \bar{\gamma}\right)\left\langle T_{n} y_{n}-z, \gamma f(z)-A z\right\rangle \\
& \leq\left\{1-\alpha_{n}\left(2 \bar{\gamma}-\alpha_{n} \bar{\gamma}^{2}-2 \gamma \alpha+2 \alpha_{n} \bar{\gamma} \gamma \alpha\right)\right\}\left\|x_{n}-z\right\|^{2}+\alpha_{n} \sigma_{n}
\end{aligned}
$$

where $\sigma_{n}=\alpha_{n}\left\|\gamma f\left(x_{n}\right)-A z\right\|^{2}+2 \beta_{n}\left\langle x_{n}-z, \gamma f(z)-A z\right\rangle+2\left(1-\beta_{n}-\alpha_{n} \bar{\gamma}\right)\left\langle T_{n} y_{n}-z, \gamma f(z)-A z\right\rangle$. By (3.47), (i) and (iii), we get lim $\sup _{n \rightarrow \infty} \sigma_{n} \leq 0$. Applying Lemma 2.2 to (3.48) we conclude that $x_{n} \rightarrow z$. This completes the proof.

Using Theorem 3.1, we obtain the following corollaries.

Corollary 3.2. Let $C$ be a nonempty closed convex subset of a real Hilbert Space H. Let $\phi$ be a bifunction of $C \times C$ into real numbers $\mathbb{R}$ satisfying (A1)-(A5). Let $f$ be a contraction of $C$ into itself 
with coefficient $\alpha \in(0,1)$ and let $T: C \rightarrow C$ be a nonexpansive mapping such that $\Theta:=F(T) \cap$ $V I(C, B) \cap E P(\phi) \neq \emptyset$. Let $B$ be a $\beta$-inverse-strongly monotone mapping of $C$ into $H$. Let $\left\{x_{n}\right\},\left\{y_{n}\right\}$ and $\left\{u_{n}\right\}$ be sequences generated by $x_{0} \in C, u_{n} \in C$ and

$$
\begin{gathered}
\phi\left(u_{n}, y\right)+\frac{1}{r_{n}}\left\langle y-u_{n}, u_{n}-x_{n}\right\rangle \geq 0, \quad \forall y \in C, \\
y_{n}=P_{C}\left(u_{n}-\lambda_{n} B u_{n}\right), \\
x_{n+1}=\alpha_{n} \gamma f\left(x_{n}\right)+\beta_{n} x_{n}+\left(1-\beta_{n}-\alpha_{n}\right) T y_{n}, \quad \forall n \geq 0,
\end{gathered}
$$

where $\left\{\alpha_{n}\right\},\left\{\beta_{n}\right\} \subset(0,1),\left\{\lambda_{n}\right\} \subset(0,2 \beta)$, for all $n \geq 0$ satisfy the condition (i), (iii)-(v). Then, $\left\{x_{n}\right\}$ converges strongly to $z \in \Theta$ where $z=P_{\Theta} z$.

Proof. Taking $T^{i}=T$ for $i=0,1, \ldots, n, \delta_{n}=0, A=I$ and $\varphi \equiv 0$ in Theorem 3.1, we can conclude the desired conclusion easily.

Corollary 3.3. Let $C$ be a nonempty closed convex subset of a real Hilbert Space H. Let $\phi$ be a bifunction of $C \times C$ into real numbers $\mathbb{R}$ satisfying (A1)-(A5) and let $T: C \rightarrow C$ be a nonexpansive mapping such that $\Theta:=F(T) \cap \operatorname{VI}(C, B) \cap E P(\phi) \neq \emptyset$. Let $B$ be a $\beta$-inverse-strongly monotone mapping of $C$ into $H$. Let $\left\{x_{n}\right\},\left\{y_{n}\right\}$ and $\left\{u_{n}\right\}$ be sequences generated by $x_{0} \in C, u_{n} \in C$ and

$$
\begin{gathered}
\phi\left(u_{n}, y\right)+\frac{1}{r_{n}}\left\langle y-u_{n}, u_{n}-x_{n}\right\rangle \geq 0, \quad \forall y \in C, \\
y_{n}=P_{C}\left(u_{n}-\lambda_{n} B u_{n}\right), \\
x_{n+1}=\alpha_{n} v+\beta_{n} x_{n}+\left(1-\beta_{n}-\alpha_{n}\right) T y_{n}, \quad \forall n \geq 0,
\end{gathered}
$$

where $\left\{\alpha_{n}\right\},\left\{\beta_{n}\right\} \subset(0,1),\left\{\lambda_{n}\right\} \subset(0,2 \beta)$, for all $n \geq 0$ satisfy the condition (i), (iii)-(v). Then, $\left\{x_{n}\right\}$ converges strongly to $z \in \Theta$, where $z=P_{\Theta} z$.

Proof. Taking $\gamma=1$ and $f(x)=v$, for all $x \in C$ in Corollary 3.2, we can conclude the desired conclusion easily.

\section{Applications to Optimization Problem}

Let $H$ be a real Hilbert space, $C$ a nonempty closed convex subset of $H, A$ a strongly positive linear bounded operator on $H$ with a constant $\bar{\gamma}>0$, and $T: C \rightarrow C$ be a nonexpansive mapping. In this section we will utilize the results present in section main results to study the following optimization problem:

$$
\min _{x \in F(T)} \frac{1}{2}\langle A x, x\rangle-h(x)
$$

where $F(T)$ is the set of fixed points of $T$ in $C$ and $h$ is a potential function for $\gamma f$ (i.e., $h^{\prime}(x)=$ $\gamma f(x)$ for $x \in H)$. We have the following theorem. 
Theorem 4.1. Let $C$ be a nonempty closed convex subset of a real Hilbert Space H. Let $T: C \rightarrow C$ be a nonexpansive mappings and let $f$ be a contraction of $C$ into itself with coefficient $\alpha \in(0,1)$. Let $A$ be a strongly positive linear bounded operator on $H$ with coefficient $\bar{\gamma}>0$ and $0<\gamma<\bar{\gamma} / \alpha$. Let $\left\{\alpha_{n}\right\},\left\{\beta_{n}\right\} \subset(0,1)$ satisfying condition (i) and (iii) in Theorem 3.1. If $F(T)$ is a nonempty compact subset of $C$, then for each $n \geq 0$ there is a unique $x_{n} \in C$ such that

$$
x_{n+1}=\alpha_{n} \gamma f\left(x_{n}\right)+\beta_{n} x_{n}+\left(\left(1-\beta_{n}\right) I-\alpha_{n} A\right) T x_{n}, \quad \forall n \geq 0,
$$

and the sequence $\left\{x_{n}\right\}$ converges strongly to some point $z \in F(T)$, which solves the following optimization problem (4.1).

Proof. Taking $\phi \equiv 0, B \equiv 0$ in Corollary 3.2, we get $P_{C}=I$ and we also have $y_{n}=x_{n}$. Hence the sequence $\left\{x_{n}\right\}$ converges strongly to some point $z \in F(T)$ which is the unique solution of the following variational inequality:

$$
\langle(A-\gamma f) z, x-z\rangle \geq 0, \quad x \in F(T) .
$$

Since $T$ is nonexpansive, then $F(T)$ is convex. Again by the assumption that $F(T)$ is compact, therefore, it is a compact and convex subset of $C$, and

$$
\frac{1}{2}\langle A x, x\rangle-h(x): C \longrightarrow R
$$

is a continuous mapping. By virtue of the well-know Weierstrass theorem, there exists a point $z^{*} \in F(T)$ which is a minimal point of optimization problem (4.1). As is know to all, (4.3) is the optimality necessary condition (see $\mathrm{Xu}[31]$ ) for the optimization problem (4.1). Then, we also have

$$
\left\langle(A-\gamma f) z^{*}, x-z^{*}\right\rangle \geq 0, \quad x \in F(T)
$$

Since $z$ is the unique solution of (4.3), therefor, $z=z^{*}$. This complete the proof of Theorem 4.1.

\section{Acknowledgments}

The authors would like to thank the Faculty of science KMUTT. Poom Kumam was supported by the Thailand Research Fund and the Commission on Higher Education under Grant no. MRG5380044.

\section{References}

[1] L.-C. Ceng and J.-C. Yao, "A hybrid iterative scheme for mixed equilibrium problems and fixed point problems," Journal of Computational and Applied Mathematics, vol. 214, no. 1, pp. 186-201, 2008.

[2] R. S. Burachik, J. O. Lopes, and G. J. P. Da Silva, "An inexact interior point proximal method for the variational inequality problem," Computational \& Applied Mathematics, vol. 28, no. 1, pp. 15-36, 2009. 
[3] E. Blum and W. Oettli, "From optimization and variational inequalities to equilibrium problems," The Mathematics Student, vol. 63, no. 1-4, pp. 123-145, 1994.

[4] S. D. Flåm and A. S. Antipin, "Equilibrium programming using proximal-like algorithms," Mathematical Programming, vol. 78, no. 1, pp. 29-41, 1997.

[5] P. Kumam, "Strong convergence theorems by an extragradient method for solving variational inequalities and equilibrium problems in a Hilbert space," Turkish Journal of Mathematics, vol. 33, no. 1, pp. 85-98, 2009.

[6] P. Kumam, "A hybrid approximation method for equilibrium and fixed point problems for a monotone mapping and a nonexpansive mapping," Nonlinear Analysis: Hybrid Systems, vol. 2, no. 4, pp. 1245-1255, 2008.

[7] P. Kumam, "A new hybrid iterative method for solution of equilibrium problems and fixed point problems for an inverse strongly monotone operator and a nonexpansive mapping," Journal of Applied Mathematics and Computing, vol. 29, no. 1-2, pp. 263-280, 2009.

[8] P. Kumam and C. Jaiboon, "A new hybrid iterative method for mixed equilibrium problems and variational inequality problem for relaxed cocoercive mappings with application to optimization problems," Nonlinear Analysis: Hybrid Systems, vol. 3, no. 4, pp. 510-530, 2009.

[9] P. Kumam and P. Katchang, "A viscosity of extragradient approximation method for finding equilibrium problems, variational inequalities and fixed point problems for nonexpansive mappings," Nonlinear Analysis: Hybrid Systems, vol. 3, no. 4, pp. 475-486, 2009.

[10] A. Moudafi and M. Théra, "Proximal and dynamical approaches to equilibrium problems," in IllPosed Variational Problems and Regularization Techniques, vol. 477 of Lecture Notes in Econom. and Math. Systems, pp. 187-201, Springer, Berlin, Germany, 1999.

[11] Z. Wang and Y.Su, "Strong convergence theorems of common elements for equilibrium problems and fixed point problems in Banach paces," Journal of Applied Mathematics \& Informatics, vol. 28, no. 3-4, pp. 783-796, 2010.

[12] R. Wangkeeree and R. Wangkeeree, "Strong convergence of the iterative scheme based on the extragradient method for mixed equilibrium problems and fixed point problems of an infinite family of nonexpansive mappings," Nonlinear Analysis: Hybrid Systems, vol. 3, no. 4, pp. 719-733, 2009.

[13] Y. Yao, M. A. Noor, S. Zainab, and Y.-C. Liou, "Mixed equilibrium problems and optimization problems," Journal of Mathematical Analysis and Applications, vol. 354, no. 1, pp. 319-329, 2009.

[14] Y. Yao, Y. J. Cho, and R. Chen, "An iterative algorithm for solving fixed point problems, variational inequality problems and mixed equilibrium problems," Nonlinear Analysis: Theory, Methods $\mathcal{E}$ Applications, vol. 71, no. 7-8, pp. 3363-3373, 2009.

[15] J.-C. Yao and O. Chadli, "Pseudomonotone complementarity problems and variational inequalities," in Handbook of Generalized Convexity and Generalized Monotonicity, vol. 76 of Nonconvex Optim. Appl., pp. 501-558, Springer, New York, NY, USA, 2005.

[16] L. C. Zeng, S. Schaible, and J. C. Yao, "Iterative algorithm for generalized set-valued strongly nonlinear mixed variational-like inequalities," Journal of Optimization Theory and Applications, vol. 124, no. 3, pp. 725-738, 2005.

[17] G. Marino and H.-K. Xu, "A general iterative method for nonexpansive mappings in Hilbert spaces," Journal of Mathematical Analysis and Applications, vol. 318, no. 1, pp. 43-52, 2006.

[18] T. Shimizu and W. Takahashi, "Strong convergence to common fixed points of families of nonexpansive mappings," Journal of Mathematical Analysis and Applications, vol. 211, no. 1, pp. 71-83, 1997.

[19] S. Plubtieng and R. Punpaeng, "A general iterative method for equilibrium problems and fixed point problems in Hilbert spaces," Journal of Mathematical Analysis and Applications, vol. 336, no. 1, pp. 455$469,2007$.

[20] J.-W. Peng and J.-C. Yao, "Strong convergence theorems of iterative scheme based on the extragradient method for mixed equilibrium problems and fixed point problems," Mathematical and Computer Modelling, vol. 49, no. 9-10, pp. 1816-1828, 2009.

[21] Y. Su, M. Shang, and X. Qin, "An iterative method of solution for equilibrium and optimization problems," Nonlinear Analysis: Theory, Methods \& Applications, vol. 69, no. 8, pp. 2709-2719, 2008.

[22] R. Wangkeeree, "An extragradient approximation method for equilibrium problems and fixed point problems of a countable family of nonexpansive mappings," Fixed Point Theory and Applications, vol. 2008, Article ID 134148, 17 pages, 2008.

[23] Y.-C. Liou and Y. Yao, "Iterative algorithms for nonexpansive mappings," Fixed Point Theory and Applications, vol. 2008, Article ID 384629, 10 pages, 2008.

[24] Y. Yao, Y.-C. Liou, and J.-C. Yao, “Convergence theorem for equilibrium problems and fixed point 
problems of infinite family of nonexpansive mappings," Fixed Point Theory and Applications, vol. 2007, Article ID 64363, 12 pages, 2007.

[25] Z. Opial, "Weak convergence of the sequence of successive approximations for nonexpansive mappings," Bulletin of the American Mathematical Society, vol. 73, pp. 591-597, 1967.

[26] R. T. Rockafellar, "On the maximality of sums of nonlinear monotone operators," Transactions of the American Mathematical Society, vol. 149, pp. 75-88, 1970.

[27] H.-K. Xu, "Viscosity approximation methods for nonexpansive mappings," Journal of Mathematical Analysis and Applications, vol. 298, no. 1, pp. 279-291, 2004.

[28] M. O. Osilike and D. I. Igbokwe, "Weak and strong convergence theorems for fixed points of pseudocontractions and solutions of monotone type operator equations," Computers $\mathcal{E}$ Mathematics with Applications, vol. 40, no. 4-5, pp. 559-567, 2000.

[29] T. Suzuki, "Strong convergence of Krasnoselskii and Mann's type sequences for one-parameter nonexpansive semigroups without Bochner integrals," Journal of Mathematical Analysis and Applications, vol. 305, no. 1, pp. 227-239, 2005.

[30] R. E. Bruck, "On the convex approximation property and the asymptotic behavior of nonlinear contractions in Banach spaces," Israel Journal of Mathematics, vol. 38, no. 4, pp. 304-314, 1981.

[31] H. K. Xu, "An iterative approach to quadratic optimization," Journal of Optimization Theory and Applications, vol. 116, no. 3, pp. 659-678, 2003. 